OPEN

SUBJECT AREAS:

STEROLS

INFLAMMATION

Received

9 October 2014

Accepted

16 February 2015

Published

25 March 2015

Correspondence and requests for materials should be addressed to

I.B. (ilaria.bellezza@ unipg.it)

\section{The loss of Tm7sf gene accelerates skin papilloma formation in mice}

\author{
I. Bellezza, L. Gatticchi, R. del Sordo, M. J. Peirce, A. Sidoni, R. Roberti \& A. Minelli
}

Dipartimento di Medicina Sperimentale, Università di Perugia, Polo Unico Sant'Andrea delle Fratte, p.le Gambuli, Perugia, 06132; Italia.

The 3 $\beta$-hydroxysterol $\Delta 14$-reductase, encoded by the $T m 7 s f 2$ gene, is an enzyme involved in cholesterol biosynthesis. Cholesterol and its derivatives control epidermal barrier integrity and are protective against environmental insults. To determine the role of the gene in skin cholesterol homeostasis, we applied 12-o-tetradecanoylphorbol-13-acetate (TPA) to the skin of $T m 7 s f 2^{+/+}$and $T m 7 s f 2^{-1}$ mice. TPA increased skin cholesterol levels by inducing de novo synthesis and up-take only in $T m 7 s f 2^{+/+}$mouse, confirming that the gene maintains cholesterol homeostasis under stress conditions. Cholesterol sulfate, one of the major players in skin permeability, was doubled by TPA treatment in the skin of wild-type animals but this response was lost in $T m 7 s f 2^{-1-}$ mice. The expression of markers of epidermal differentiation concomitant with farnesoid-X-receptor and p38 MAPK activation were also disrupted in $\mathrm{Tm} 7 \mathrm{sf} 2^{-1-}$ mice. We then subjected $T m 7 s f 2^{+/+}$and $T m 7 s f 2^{-/}$mice to a classical two-stage skin carcinogenesis protocol. We found that the loss of $T m 7 s f 2$ increased incidence and multiplicity of skin papillomas. Interestingly, the null genotype showed reduced expression of nur77, a gene associated with resistance to neoplastic transformation. In conclusion, the loss of $T m 7 s f 2$ alters the expression of proteins involved in epidermal differentiation by reducing the levels of cholesterol sulfate.

$\mathrm{n}$ animal tissues, cholesterol (cholest-5-en-3 $\beta$-ol) is the most abundant member of a family of polycyclic compounds known as sterols. Cholesterol biosynthesis involves numerous enzymes either cytosolic or endo-

plasmic reticulum-resident. The 3 $\beta$-hydroxysterol $\Delta 14$-reductase (C14SR, EC 1.3.1.70), encoded by the Tm7sf2 gene, residing in the endoplasmic reticulum and recently found in the nucleous ${ }^{1}$, reduces the C14$\mathrm{C} 15$ of unsaturated sterol intermediates ${ }^{2}$ in cholesterol biosynthesis. In order to define the in vivo functions of this protein, Tm7sf2-null mice were generated; these mice develop normally, are fertile and show no obvious abnormalities $^{3}$. However, following tunicamycin treatment, Tm7sf2-null mice fail to increase hepatic cholesterol levels ${ }^{4}$, indicating that is essential for cholesterol biosynthesis, at least under stress conditions.

Cholesterol levels are of particular importance to the physiology and pathophysiology of the skin. For example, maintenance of cholesterol homeostasis in keratinocytes/epidermis is required to form lamellar bodies ${ }^{5-6}$. These fuse together in the stratum corneum to generate a continuous layer of lipids to form an impermeable barrier ${ }^{7-8}$. Disruption of this barrier up-regulates cholesterol synthesis ${ }^{9}$, along with levels of the receptors responsible for cholesterol uptake such as the low density lipoprotein receptor (Ldlr) ${ }^{10}$. On the other hand, inhibition of cholesterol synthesis perturbs barrier function ${ }^{11}$. Indeed deficiency in cholesterol synthesis largely accounts for the barrier abnormality in aged epidermis ${ }^{12-13}$. Beyond the barrier function of the epidermis, cholesterol synthesis is believed to be protective against a range of inflammatory and chemical insults to the skin ${ }^{14}$. Finally, cholesterol is the precursor of an important bioregulatory molecule in keratinocytes, cholesterol sulfate (CS), which regulates corneocyte desquamation and cohesion ${ }^{15}$ and keratinocyte differentiation ${ }^{16}$.

Given these important structural and protective roles of cholesterol in the skin, we used the tumour-promoting phorbol ester, which has been shown to disrupt sterol metabolism of mouse $\operatorname{skin}^{17}$, to define the role of Tm $7 \mathrm{sf} 2$ in skin cholesterol homeostasis and susceptibility to tumour growth.

\section{Results}

Tm7sf2 gene drives cholesterol accumulation after TPA exposure. Cholesterol and/or its derivatives may play a direct role in epidermal homeostasis and TPA is known to change the metabolism of sterols in mouse skin ${ }^{17}$. To analyse the role of Tm7sf2 gene in cholesterol epidermal homeostasis we topically treated Tm7sf $2^{+/+}$and Tm7sf $2^{-1-}$ mice with $20 \mathrm{nmol}$ of TPA and determined cholesterol levels at $72 \mathrm{~h}$. We found that the skin of $\mathrm{Tm} 7 \mathrm{sf} 2^{+/+}$and $\mathrm{Tm} 7 \mathrm{sf} 2^{-/-}$mice contained comparable basal amounts of cholesterol $(0.88 \pm 0.02$ vs. $0.73 \pm$ 

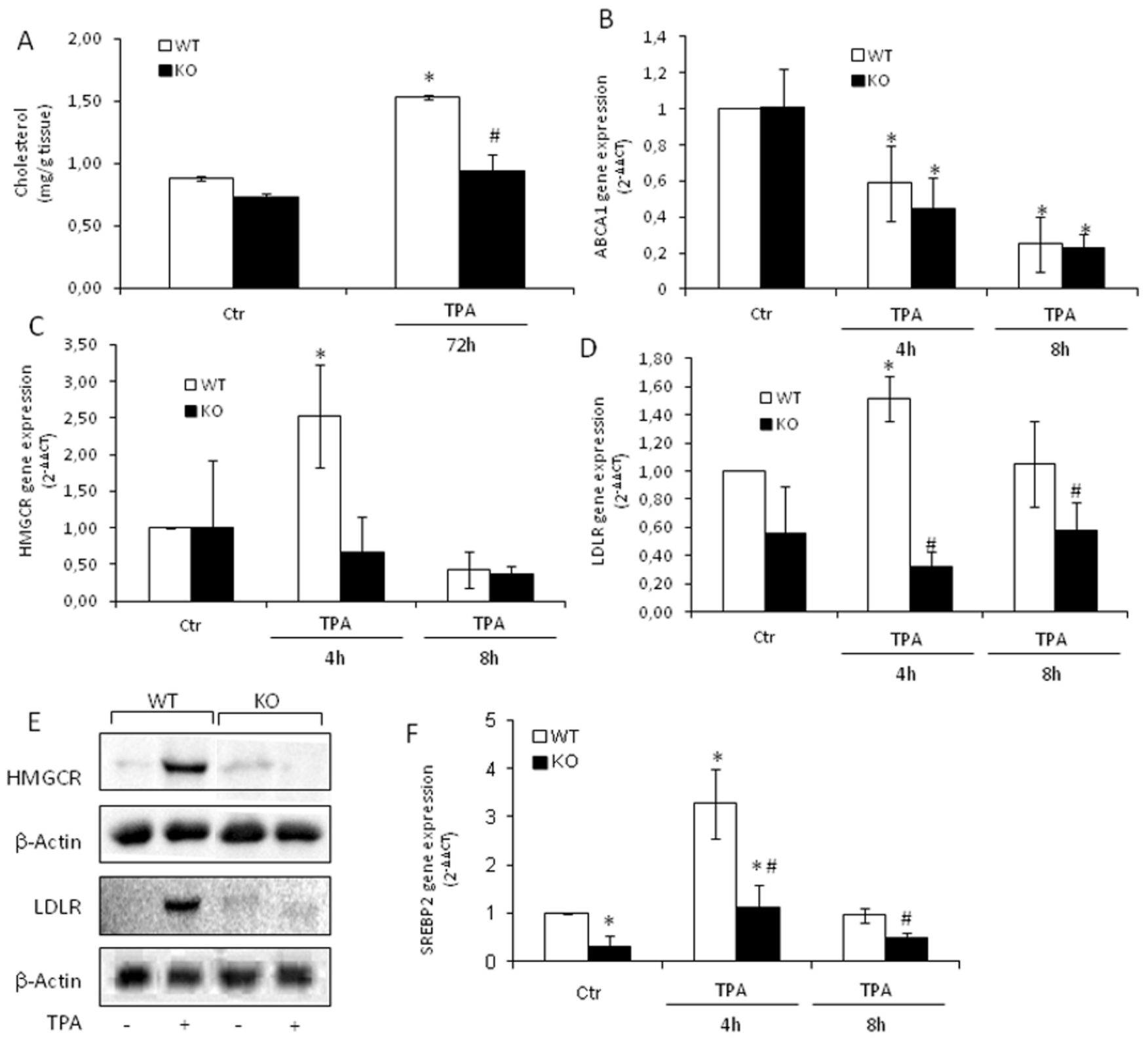

Figure $1 \mid$ Tm7sf2 gene drives cholesterol accumulation after TPA exposure. WT and Tm7sf2 KO mice were subjected to a single skin topical application of TPA and sacrificed at the indicated time. The skin was removed and used to determine: (A) cholesterol levels by TLC analysis ( $\mathrm{n}=3$ ); expression of (B) Abca1, (C) Hmgcr, (D) Ldlr, (F) SREB-2 by Real Time RT- PCR analyses. Expression of each gene was normalized to Gapdh and reported as $2^{-\Delta \Delta C t}$. Relative mRNA level of WT untreated mice skin was assumed as 1 . Results are given as mean \pm S.D. $(n=4),{ }^{*} p<0.05$ vs. untreated WT, \# $p<0.05$ vs. the respective WT. (E) Whole skin lysates (pooled samples from $n=5$ mice) were analyzed by western blotting with the indicated antibodies. Anti $\beta$-actin was used as loading control.

$0.01 \mathrm{mg} \mathrm{g}$ of tissue ${ }^{-1}$ ) and that TPA increased cholesterol levels only in the skin of Tm7sf2 $2^{+/+}$mice (Fig. 1A). It is to note that treatment with acetone did not affect skin cholesterol levels (Fig. S1 A and B).

Cholesterol accumulation may be due to: (i) increased de novo synthesis via up-regulation of 3-hydroxy-3-methylglutaryl-CoA reductase (Hmgcr), (ii) increased uptake of circulating cholesterol via the low-density lipoprotein receptor (Ldlr), and (iii) decreased cellular cholesterol efflux via the ATP-binding cassette transporter (Abca1). We found that TPA induced a decrease in Abcal expression in both genotypes (Figure 1B), whereas it induced a significant increase in the mRNA levels of both Hmgcr and Ldlr only in the skin of Tm7sf $2^{+/+}$mice (Fig. $1 \mathrm{C}$ and D). Results were confirmed by Western blotting at $8 \mathrm{~h}$ TPA exposure (Fig. 1E). Consistent with these findings, sterol regulatory element binding protein 2 (SREBP2 ), the key transcription factor that induces the expression of both
Ldlr and $\mathrm{Hmgcr}^{18}$, was found markedly increased at $4 \mathrm{~h}$ TPA exposure in the skin of Tm7sf2 ${ }^{+/+}$mice (Fig. 1F). Interestingly, SREBP-2 gene expression was lower in Tm7sf $2^{--}$mice in basal conditions and was significantly up-regulated by TPA (Fig. $1 \mathrm{~F}$ ), whereas its activation was induced by TPA independently of the genotype (Fig. S1C). Data indicate that the Tm7sf2 gene regulates cholesterol homeostasis by inducing de novo synthesis and up-take from circulation after TPA treatment.

Tm7sf2 gene regulates cholesterol sulfate homeostasis. Skin cholesterol is converted by cholesterol sulfotransferases (Sults) into cholesterol sulfate (CS) involved in keratinocyte differentiation and development of the epidermal barrier ${ }^{19}$. To assess whether Tm7sf2 can affect skin CS levels, we analyzed CS levels at $72 \mathrm{~h}$ TPA treatment (Fig. 2A). We found that the skin of Tm $7 \mathrm{sf} 2^{+/+}$and Tm7sf $2^{-1-}$ mice 
A
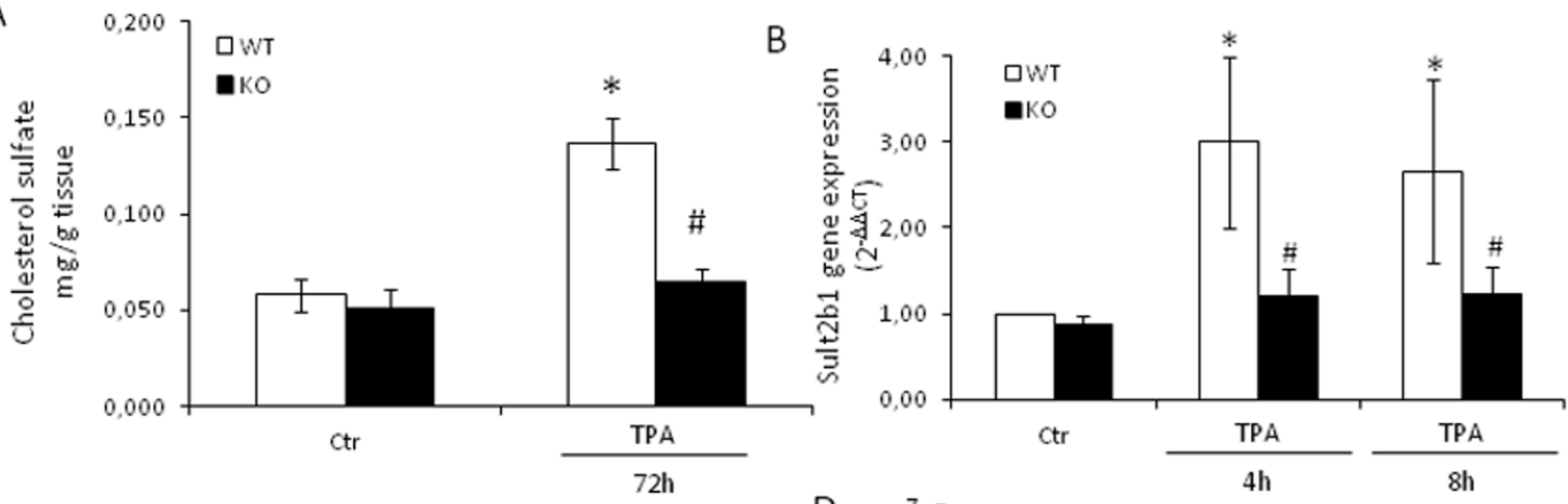

C
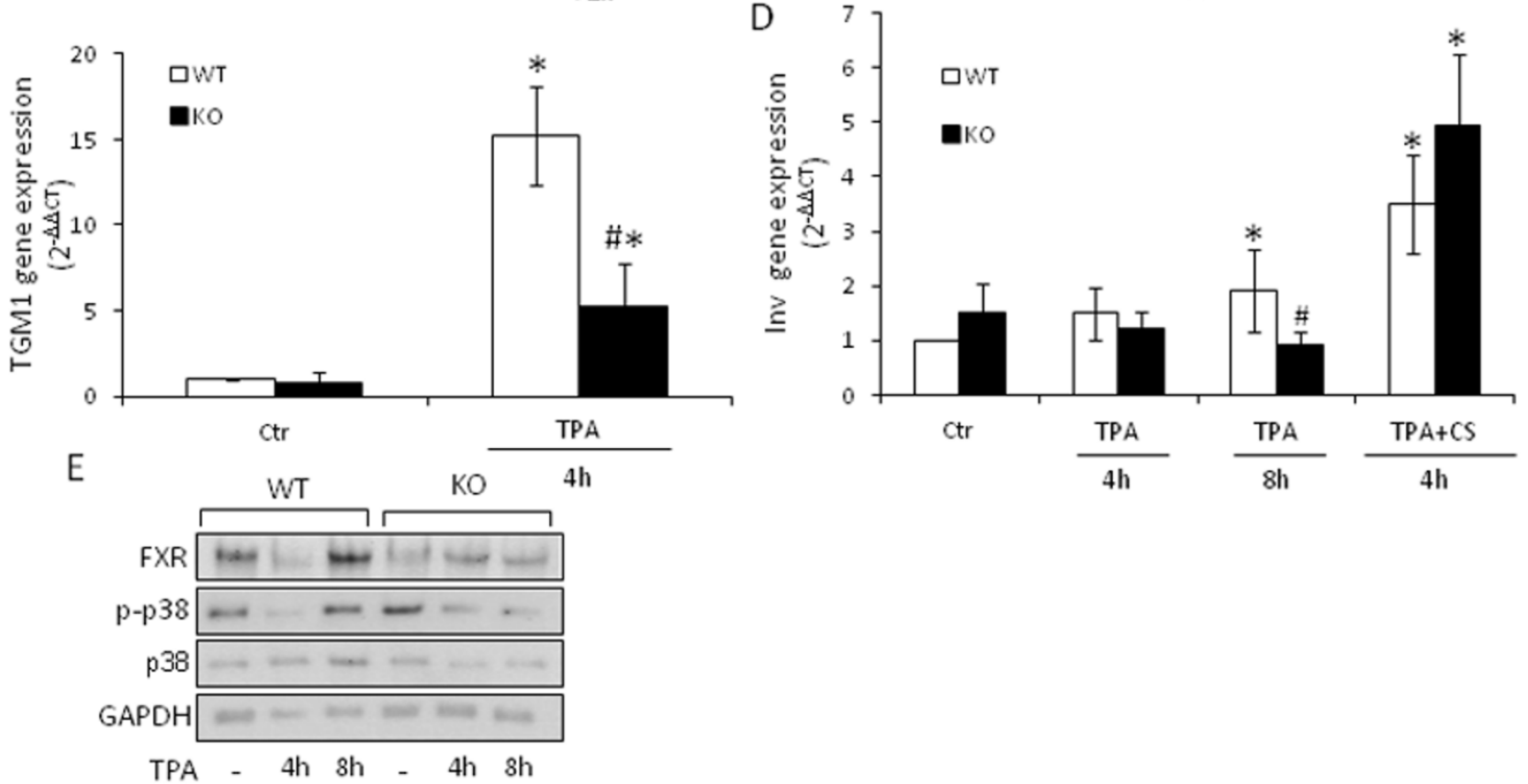

Figure $2 \mid \mathrm{Tm} 7 \mathrm{sf} 2$ gene regulates cholesterol sulfate homeostasis. WT and Tm7sf2 KO mice were subjected to a single topical application of TPA and sacrificed at the indicated time. The skin was used to determine: (A) cholesterol sulfate levels by TLC analysis ( $\mathrm{n}=3$ ); expression of (B) Sult2B1, (C) Tgm1, and (D) Inv by Real Time RT- PCR analyses. When cholesterol sulfate was used, mice of both genotypes were pre-treated with $820 \mu \mathrm{mol}$ of cholesterol sulfate $10 \mathrm{~min}$ before the treatment with TPA for $4 \mathrm{~h}$. Expression of each gene was normalized to Gapdh and reported as $2^{-\Delta \Delta \mathrm{Ct}}$. Relative mRNA level of WT untreated mice skin was assumed as 1 . Results are given as mean \pm S.D. $(n=4),{ }^{*} \mathrm{p}<0.05$ vs. untreated WT, \# $\mathrm{p}<0.05$ vs. the respective WT. (E) Whole skin lysates (pooled samples from $n=5$ mice) were analyzed by Western Blotting with the indicated antibodies. Anti $\beta$-actin was used as loading control.

contained comparable basal amounts of CS $(0.082 \pm 0.02$ vs. $0.068 \pm$ $0.01 \mathrm{mg} g$ of tissue $\mathrm{e}^{-1}$ ) and that TPA induced a doubling in CS levels only in the Tm7sf $2^{+/+}$mouse skin (Fig. $2 \mathrm{~A}$ ).

Sult2B1 is the main isozyme of the Sult family active in sulfonating cholesterol $^{20}$. Thus, to determine whether the loss of the Tm7sf2 gene can cause decreased expression of the Sult2B1 gene, we analyzed its expression up to $8 \mathrm{~h}$ TPA treatment and found that TPA induced a significant increase in Sult2B1 mRNA levels only in the Tm7sf $2^{+/+}$ mouse skin (Fig. 2B). These data indicate that the Tm7sf2 gene regulates CS biosynthesis by increasing cholesterol levels and the expression of an enzyme involved in cholesterol sulfonation.

CS is implicated in the regulation of the early and late stages of keratinocyte differentiation ${ }^{16,21-22}$. We surmised that the markers of keratinocyte differentiation could be altered by the loss of the Tm7sf2 gene. Therefore, we treated mice of both genotypes with TPA and analysed the mRNA levels of transglutaminase 1 (Tgm1) and involucrin (Inv). We found that TPA exposure significantly increased Tgm 1 and Inv mRNA levels only in Tm7sf2 $2^{+/+}$mouse skin (Fig. 2C and D) suggesting that the lack of Tm7sf2 alters the expression of markers of differentiation after TPA exposure.

To confirm the role of CS in Inv mRNA induction, we pre-treated mice of both genotypes with $820 \mu \mathrm{mol}$ of CS $10 \mathrm{~min}$ prior to TPA treatment (Fig. 2D). We found that exogenous CS, while not affecting the TPA-induced HMGCR expression (Fig. S2), increased the Inv mRNA expression independently of the genotype, confirming the pivotal role for Tm7sf 2 in keratinocyte differentiation.

The nuclear receptor Farnesoid-X-receptor (FXR) and p38 MAP kinase are deeply involved in skin development and functionality, the former by accelerating the formation of a mature stratum corneum $^{23}$, the latter by up-regulating Inv expression ${ }^{24}$. Thus, we analysed by western blotting the activation of p38 and the expression of FXR (Fig. 2E) and found that TPA failed to induce their activation/expression in $\mathrm{Tm} 7 \mathrm{sf} 2^{-/-}$mouse skin, indicating that $\mathrm{Tm} 7 \mathrm{sf} 2$ regulates multiple signalling pathways responsible for epidermal differentiation. 
Tm7sf 2 controls papilloma incidence and multiplicity. Cholesterol levels have been linked to keratinocyte proliferation. Indeed, cholesterol depletion, acting through the EGFR, activates the extracellular signal-regulated kinases (ERK) $1 / 2^{25}$. To verify whether the loss of Tm7sf2 impairs keratinocyte proliferation, we treated the skin of $\mathrm{Tm} 7 \mathrm{sf} 2^{+/+}$and $\mathrm{Tm} 7 \mathrm{sf} 2^{-/-}$mice topically with a single dose of TPA and measured epidermal thickness at $24 \mathrm{~h}$. We found that the TPA challenge induced a significant increase in epidermal thickness only in Tm7sf $2^{-1-}$ mice (Fig. 3A). We verified by western blotting the activation of ERK1/2, a well-known driver of cell proliferation in this model ${ }^{26}$. We found increased phosphorylation of ERK1/2 only in the skin of Tm7sf $2^{-1-}$ mice (Fig. 3B). Keratinocyte growth factor (KGF) via ERK $1 / 2$ activation is responsible for epidermal cell proliferation ${ }^{27}$. Consistent with our results, we found that TPA exposure caused an increase in KGF expression in the skin of $\mathrm{Tm} 7 \mathrm{sf} 2^{-1-}$ mice at $8 \mathrm{~h}$ TPA exposure (Fig 3C). Results indicate that Tm7sf2 gene is involved in the control of skin proliferation after TPA challenge. To better elucidate the role of the Tm7sf2 gene in skin papilloma formation after TPA treatment, we analyzed the expression of the orphan nuclear receptor Nur77, linked to resistance to neoplastic transformation in the $\operatorname{skin}^{28}$. We found that a $4 \mathrm{~h}$ TPA exposure caused a marked increase in Nur77 expression only in the skin of Tm7sf $2^{+/+}$mice (Fig. 3D).

By mimicking the multistage nature of human cancer development $^{29}$, the DMBA/TPA-induced two-stage skin carcinogenesis serves as a well-characterized in vivo mouse model for epithelial neoplasia. To assess the role of Tm7sf2 in skin tumourigenesis, we treated mice of both genotypes with DMBA/TPA. We observed that both genotypes developed papillomas with similar histological features (Fig. 4A). The first detectable tumour $(>2 \mathrm{~mm})$ appeared after 9 weeks of treatment in the null genotype and after 11 weeks in Tm $7 \mathrm{sf} 2^{+1+}$ mice. By week 15, 30\% of $\mathrm{Tm} 7 \mathrm{sf} 2^{+1+}$ and $55 \%$ of Tm7sf $2^{-1-}$ mice showed papillomas (Fig 4B). Even tumour multiplicity was higher in $\mathrm{Tm} 7 \mathrm{sf} 2^{-1-}$ animals and this difference reached statistical significance after 15 weeks of treatment (Fig. 4C). By week 19, papillomas developed in Tm7sf $2^{-1-}$ mice were greater in size (17 $\pm 10 \mathrm{~mm}^{2}$ in WT vs $43.2 \pm 14 \mathrm{~mm}^{2}$ in KO mice $\mathrm{p}=0.039$ ) (Fig. 4D). These results demonstrate that loss of Tm7sf2 markedly increases size, incidence and multiplicity of skin papillomas.
A

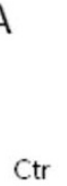

Ctr
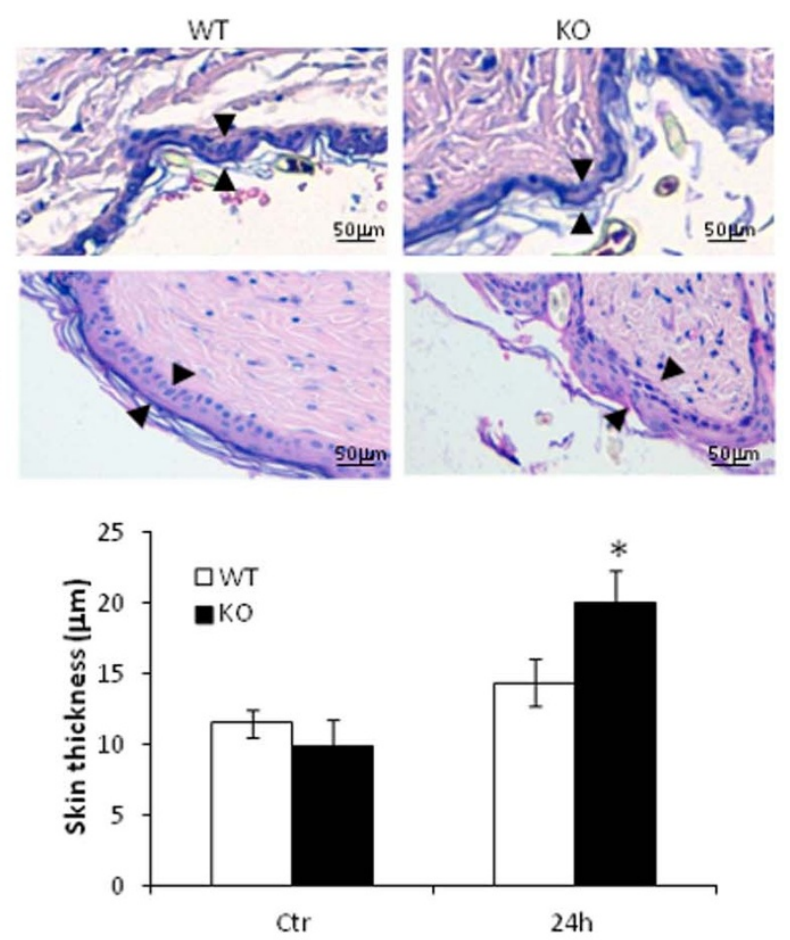

B
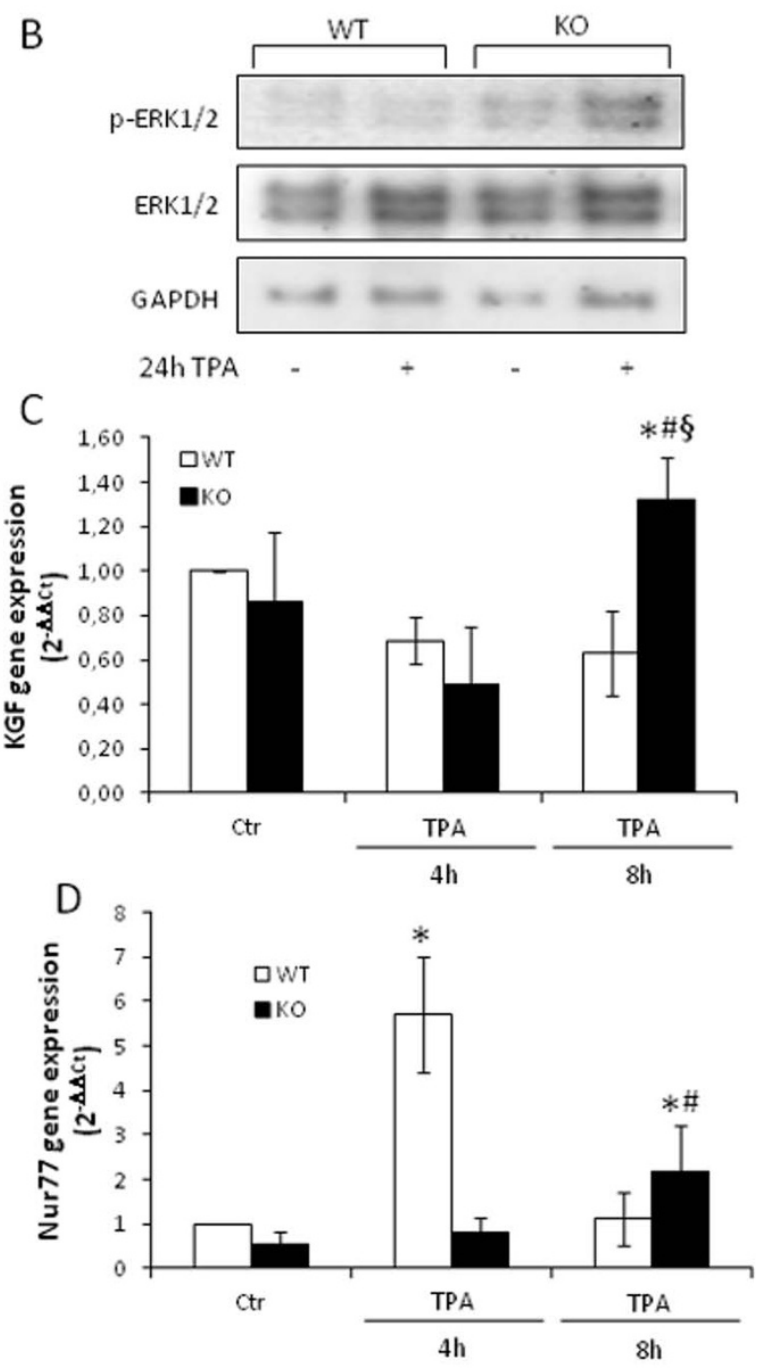

Figure $3 \mid \mathrm{Tm} 7 \mathrm{sf} 2$ controls TPA-induced epidermal hyper-proliferation. WT and Tm7sf2 KO were subjected to a single skin topical application of TPA and (A) skin architecture and epidermal thickness were analysed with H\&E staining (magnification 200X), arrows indicate epidermal thickness; quantification was performed on $\mathrm{n}=4$ skin samples, each measured in at least 3 different fields; (B) levels of phosphorylated ERK1/2 by western blotting ( $\mathrm{n}=5$ pooled samples). ${ }^{*} \mathrm{P}<0.05$ vs control mice of the respective genotype. WT and Tm7sf2 KO mice were subjected to a single topical application of TPA and sacrificed at the indicated time. The skin was used to determine the expression of (C) KGF and (D) Nur77 by Real Time PCR analyses. Expression of each gene was normalized to Gapdh and reported as $2^{-\Delta \Delta C t}$. Relative mRNA level of WT untreated mice skin was assumed as 1 . Results are given as mean \pm S.D. $(\mathrm{n}=4),{ }^{*} \mathrm{p}<0.05$ vs. untreated WT, $\# \mathrm{p}<0.05$ vs. the respective WT. 


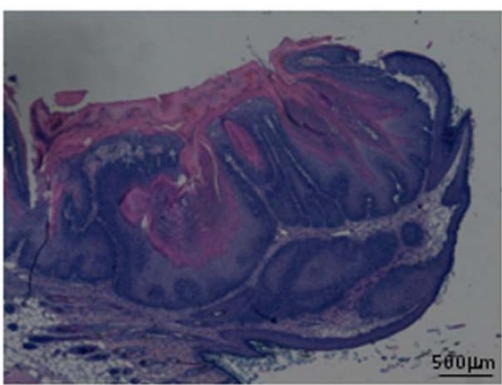

B

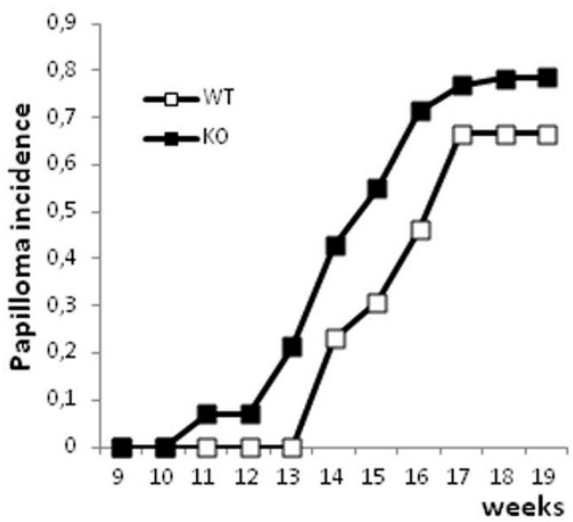

D

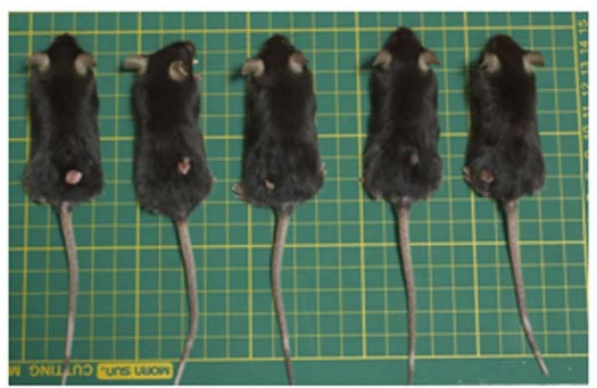

KO

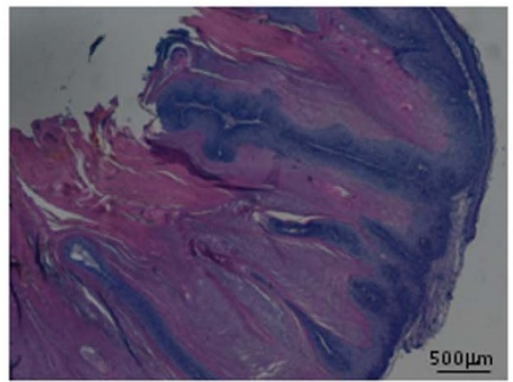

C

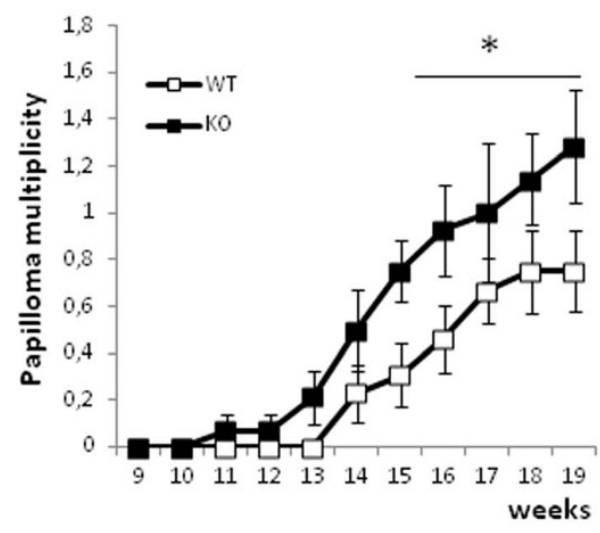

KO

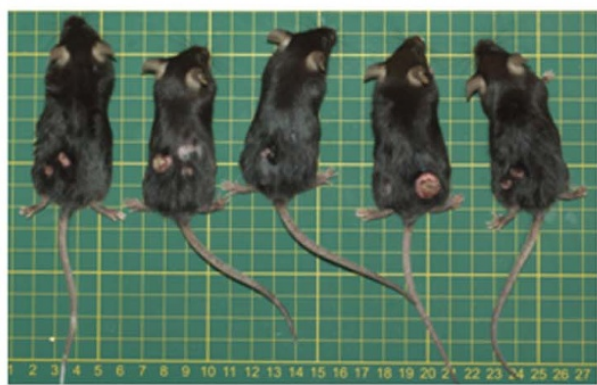

Figure $4 \mid$ The lack of Tm7sf2 increases papilloma incidence and multiplicity. Tm7sf2 WT $(n=15)$ and KO $(n=15)$ mice were topically treated with a single dose of DMBA followed by bi-weekly applications of TPA for 19 weeks. (A) H\&E staining of $4 \mu \mathrm{m}$ sections from papillomas of Tm7sf2 WT and KO mice. (B) Papilloma incidence and (C) papilloma multiplicity at the indicated times after DMBA treatment. (D) Representative images of Tm7sf2 WT and KO papilloma-bearing mice (magnification $40 \times$ ). ${ }^{*} \mathrm{p}<0.05$ vs control WT mice.

\section{Discussion}

In the present study, we found that the loss of the Tm7sf 2 gene markedly increases size, incidence and multiplicity of skin papillomas by altering skin cholesterol levels in a mouse model of skin carcinogenesis.

Consistent with previous reports ${ }^{2}, \mathrm{Tm} 7 \mathrm{sf} 2$-null mice displayed normal epidermal morphology, indicating that Tm7sf2 is not essential for epidermal homeostasis under basal conditions. This could be explained by the possible functional compensatory redundancy of the lamin $\mathrm{B}$ receptor $(\mathrm{LBR})^{2,30}$. However our data demonstrate unique roles for Tm7sf2 in the resistance to tumour development and in the control of cholesterol biosynthesis in the skin that follows TPA challenge; the up-regulation of the cholesterol biosynthetic machinery noted in wild type mice after TPA treatment was lost in the Tm7sf 2 knock out animals while tumours in the knock out animals were more numerous, larger in size and developed more rapidly. The association between tumour development and loss of
Tm7sf2 expression is consistent with published data showing reduced Tm7sf2 expression in both cancer cell lines and in primary adrenocortical tumour tissue as compared to the respective normal tissues $^{2,31-32}$.

While the mechanism by which the loss of Tm7sf2 expression promotes tumour development in the skin remains to be definitively elucidated our data offer some tantalising clues. Firstly, proliferative signals and signalling pathways, important features of tumour development ${ }^{33-34}$, appear to be exaggerated in the absence of Tm7sf2. Thus, the TPA-induced production of KGF, which can induce skin hyper-proliferation ${ }^{35}$ and activation of the ERK pathway, through which it promotes proliferation and DNA synthesis ${ }^{27}$, are both augmented in Tm7sf 2 null mice. Activation of ERK1/2 can also be caused by low cell cholesterol levels ${ }^{25,36}$. Secondly, other TPAinducible pathways associated with resistance to neoplastic transformation, such as the orphan nuclear receptor Nur $77^{28}$, are lost in the Tm7sf2 KO animals. While the target genes of this transcription 
Table 1 | List of primers

Gene name

Gene symbol

Primer sequence (F: Forward; R: Reverse)

ATP-binding cassette transporter 1

Abcal

Glycerhaldeyde 3-phosphate dehydrogenase

Gapdh

HMG-CoA reductase

Hmgcr

Involucrin

Inv

Keratinocyte growth factor

KGF

F: CCAGACGGAGCCGGAAGGGT

R: GTGCCCATGTCCTCGGGAGC

F:GCCAAATTCAACGGCACAGT

R:AGATGGTGATGGGCTTCCC

F: TGCCTGGATGGGAAGGAGTA

R: GCCTCGAGTCATCCCATCTG

F: CCCTCCTGTGAGTTTGTTTGG

R: TGAGAGGTCCCTGAACCACA

F: ACGGCTACGAGTGTGAACTG

R: GGGTCCCITTCACTTTGCCT

LDL Receptor

Ldlr

F: GGGAACATTTCGGGGTCTGT

R: AGTCTTCTGCTGCAACTCCG

Nuclear receptor subfamily 4 group A member 1

Nr4al (Nur77)

F: TGTGCTAGAAGGACTGCGGA

R: ATGGTAGGCTTGCCGAACTC

Sterol regulatory element-binding protein 2

Srebp-2

F: CAAGCACACTGATTGAGAT

Cytosolic Sulfotransferase 2B 1

Sult2B 1

R:TGGGCACGATTTAAGAAGTA

F: GCCCAGAGTGAGGCTTTTGA

R: CCTCTTCAGACGTGTCCCAG

F: CTGTTGGTCCCGTCCCAAAC

Tranglutaminase 1

$\operatorname{Tgm} 1$

R: GGACCTTCCATTGTGCTGGAG

factor in the skin are currently unknown, our data connect Tm7sf2 to both anti-proliferative and tumour resistance responses.

Cholesterol biosynthesis is essential for skin homeostasis and protection against toxic topical insults as evidenced by the abnormal cutaneous phenotypes associated with the loss of cholesterol biosynthetic enzymes ${ }^{37-42}$. Our data demonstrate a requirement for Tm7sf2 in a host of protective skin responses to TPA challenge. TPA-induced increases in skin cholesterol levels, elements of the cholesterol biosynthetic machinery (Hmgcr) and cholesterol uptake (Ldlr), as well as increased expression of the transcription factor (Srebp-2) responsible for driving expression of these genes ${ }^{18}$ detected in wild type animals, were all lost in Tm7sf $2^{-1-}$ mice. Interestingly, TPA-induced SREBP-2 activation was independent of the genotype. Nevertheless, it has been demonstrated that Nur77 regulates cholesterol levels through the suppression of LDLR and HMGCR expression ${ }^{43}$, suggesting a pivotal role for this orphan receptor in the regulation of cholesterol metabolism.

At the same time, the activation of pathways associated with the returning cholesterol biosynthesis to basal levels $8 \mathrm{~h}$ after TPA challenge, such as the p38 MAPK pathway and the nuclear receptor farnesoid-X-receptor (FXR) ${ }^{44}$, are abolished in the Tm7sf $2^{-/}$mice. This presumably reflects the failure in these animals to initiate a complex program of gene expression designed to produce a pulse of cholesterol biosynthesis in response to TPA challenge. Notably the loss of these responses in other systems (e.g. hepatocellular carcinoma) is associated with a more aggressive proliferation ${ }^{45-46}$ consistent with the enhanced papilloma development observed in our skin model. Interestingly a second, related nuclear receptor, liver X receptor (LXR) which controls expression of the cholesterol exporter protein, Abca1, appeared unaffected by loss of Tm7sf2 because Abca1 expression was down regulated equally in both genotypes in response to TPA-challenge. This suggests that at least some TPA-mediated responses are independent of Tm7sf2.

The above effects relate to the importance of cholesterol in controlling skin physiology but its derivative, cholesterol sulfate, also functions as a key controller of epidermal differentiation. CS is synthesized from cholesterol by cholesterol sulfotransferase (Sult), which, in turn, is up-regulated by inducers of epidermal differentiation such as calcium ${ }^{19,47}$. We showed that the TPA-induced increases in CS levels and in the expression of Sult observed in wild type mice was lost in Tm7sf2 null mice. This finding may relate to the observed lack of increase of FXR in the null genotype since FXR small interfering RNA (siRNA) significantly reduces Sult2a1 promoter activity $^{48}$. Thus, Tm7sf2 appears to control the level of CS by affecting both the level of cholesterol and its subsequent sulfonation by Sult.

CS drives differentiation of keratinocytes by activating protein kinase $\mathrm{C}$ leading to activation of the transcription factor activator protein-1 (AP1). This drives expression of several markers of differentiation such as Tgm1 and $\operatorname{Inv}^{21-22,49-50}$. In our experiments, we documented the expected increase in Tgm 1 and Inv expression only in the skin of Tm7sf $2^{+/+}$mice. Notably, in the case of Inv, the deficit was rescued by the addition of exogenous CS suggesting that the observed reduction was a direct result of the lack of CS in Tm7sf $2^{-1-}$ mouse skin. As expected, CS addition did not result in an increased expression of the up-stream gene Hmgcr. Since the expression of Inv is controlled by the p38 pathway ${ }^{24}$, this observation may relate to the failure of TPA to activate the $\mathrm{p} 38$ pathway in the Tm $7 \mathrm{sf} 2^{-1-}$ mice discussed above.

In conclusion, the data presented here strongly implicate Tm7sf2 in driving a host of protective responses after topical TPA challenge, centred on increased cholesterol and CS levels.

\section{Methods}

Materials. All the reagents, unless otherwise stated, were from Sigma Aldrich (St. Luis, MO). Cell culture reagents were from Life Technologies (GibcoBRL, Gaithersburg, MD).

Animals. Tm7sf2 $2^{+/+}$and Tm7sf2 $2^{-1-}$ mice, on a C57BL/6 background ${ }^{3}$ ( $>20$ backcross), were housed at the Laboratory Animal Research Centre of Perugia University. The animals were maintained at a constant temperature of $22^{\circ} \mathrm{C}, 12 \mathrm{~h}$ light/dark cycle, and fed ad libitum.

Ethics statement. All experimental procedures were carried out in accordance with European Directives, approved by the Institutional Animal Care and Use Committee of Perugia University (106/2012). Efforts were made to minimise animal stress/ discomfort.

TPA-induced inflammation in mouse skin. The shaved dorsal regions of female $\mathrm{Tm} 7 \mathrm{sf} 2^{+1+}$ and Tm7sf $2^{-1-}$ mice (6-8 weeks of age) were treated topically with $20 \mathrm{nmol}$ of TPA (12-o-tetradecanoylphorbol-13-acetate) in $100 \mu \mathrm{L}$ of acetone or with acetone alone. Mice were sacrificed by $\mathrm{CO}_{2}$ asphyxiation $4,8,24$ or $72 \mathrm{~h}$ after TPA treatment. The dorsal skin was excised, and the fat from the whole skin was removed on ice. The fat-free skin tissues were then frozen immediately in liquid nitrogen or fixed in formalin. When cholesterol sulfate was used, mice of both genotypes were pre-treated with $820 \mu \mathrm{mol}$ of cholesterol sulfate $10 \mathrm{~min}$ before TPA treatment.

Two-stage skin carcinogenesis protocol. The shaved dorsal regions of female $\mathrm{Tm} 7 \mathrm{sf} 2^{+1+}$ and Tm7sf $2^{-1-}$ mice (6-8 weeks of age) were treated topically with a single dose of $0.2 \mathrm{mmol}$ DMBA (7,12-dimethylbenz[ $\alpha]$ anthracene) dissolved in $100 \mu \mathrm{L}$ of acetone. One week later the mice were treated topically with $20 \mathrm{nmol}$ of TPA in 
$100 \mu \mathrm{L}$ of acetone $(\mathrm{n}=15)$ or acetone alone $(\mathrm{n}=5)$ twice weekly for 19 weeks. The incidence and number of tumours that were at least $2 \mathrm{~mm}$ in diameter were monitored and counted weekly. The results were expressed as the percentage of tumour-bearing mice (tumour incidence) and the average number of tumours per mouse (tumour multiplicity).

Western Blotting. Skin tissue (20\% w/v) was lysed in a boiling Laemmli sample buffer, resolved on SDS-polyacrylamide gels and transferred to nitrocellulose membranes. Membranes were probed with anti Hmgcr, anti Ldlr (Abcam, Cambridge, UK), anti phosphor-p38 (Tyr180/Tyr182) (Cell signaling technology, Danvers, MA), anti FXR, anti SREBP-2 (CRL-2545) (ATCC), anti Gapdh and anti $\beta$ actin (Santa Cruz Biotechnology, Santa Cruz, CA) which were detected using HRPbased chemiluminescence (ECL, Pierce Biotechnology, Rockford, IL). Each sample was prepared identically from five individual animals and, within each group, results were highly reproducible.

Real Time RT-PCR. Total RNA was isolated with TRI Reagent according to the manufacturer's instructions and cDNA was synthesised using iScript cDNA synthesis kit (Bio-Rad Lab, Hercules, CA). Real time PCR was performed using the iCycler iQ detection system (Bio-Rad Lab, Hercules, CA) and SYBR Green chemistry. Primers are listed in Table 1. SYBR Green RT-PCR amplifications were carried out in a 96-well plate in a $25 \mu \mathrm{L}$ reaction volume that contained $12,5 \mu \mathrm{L}$ of SYBR ${ }^{\circledR}$ Green JumpStart $^{\mathrm{TM}} \mathrm{Taq}$ ReadyMix ${ }^{\mathrm{TM}}, 400 \mathrm{nmol} \mathrm{L}^{-1}$ forward and reverse primers, and 5 to $40 \mathrm{ng}$ of cDNA. In each assay, no-template controls were included and each sample was run in triplicate. Mean of $C_{t}$ values of the samples was compared to the untreated control sample and Gapdh used as internal control. The n-fold differential ratio was expressed as $2^{-\Delta \Delta C t}$.

Histochemistry. Paraffin-embedded sections from mouse skin were collected for immunohistochemical (IHC) analysis. Briefly, tissue samples were fixed in $10 \%$ buffered formalin and embedded in paraffin. The $4 \mu \mathrm{m}$ tissue sections were stained with hematoxylin \& eosin.

Determination of cholesterol and cholesterol sulfate by TLC. Skin explants $(50 \mathrm{mg})$ were incubated overnight at $55^{\circ} \mathrm{C}$ in $0.2 \mathrm{~mL}$ of lysis buffer $\left(0.1 \mathrm{~mol} \mathrm{~L}^{-1}\right.$ Tris$\mathrm{HCl}, \mathrm{pH} 8.0,12.5 \mathrm{mmol} \mathrm{L}^{-1}$ EDTA, $0.2 \mathrm{~mol} \mathrm{~L}^{-1} \mathrm{NaCl}$, and $0.25 \mathrm{mg} \mathrm{mL}^{-1}$ proteinase $\mathrm{K})$, then lipids were extracted by the Bligh and Dyer method in the presence of $1 \mathrm{~mol}$ $\mathrm{L}^{-1} \mathrm{KCl}$ to allow full cholesterol sulfate recovery ${ }^{51}$. Cholesterol was analyzed by TLC as previously described ${ }^{3}$. CS was analyzed by TLC using diethyl ether/n-hexane/acetic acid $(45: 40: 15)$ as developing mixture. Plates were stained with $\mathrm{Cu}$-acetate/ phosphoric acid ${ }^{52}$. Images were acquired using the VersaDoc Imaging System and signals were quantified using Quantity One software (Bio-Rad, Milan, Italy) and pure cholesterol and cholesterol sulfate standards for reference.

Statistical Analysis. The results were expressed as the mean \pm SD and analyzed via analysis of variance. Differences between the treatment groups were evaluated via Student's t-test, except the differences in tumour incidence and multiplicity that were analyzed via a two-sample test for binomial proportions. Differences were considered significant at $\mathrm{p}<0.05$

1. Zuleger, N. et al. Specific nuclear envelope transmembrane proteins can promote the location of chromosomes to and from the nuclear periphery. Genome Biol. 14 R14 (2013).

2. Bennati, A. M. et al. Sterol dependent regulation of human TM7SF2 gene expression: role of the encoded 3beta-hydroxysterol Delta14-reductase in human cholesterol biosynthesis. Biochim. Biophys. Acta. 1761, 677-685 (2006).

3. Bennati, A. M. et al. Disruption of the gene encoding 3beta-hydroxysterol Deltareductase (Tm7sf2) in mice does not impair cholesterol biosynthesis. FEBS J. 275 5034-5047 (2008)

4. Bellezza, I. et al. A novel role for Tm7sf2 gene in regulating TNF $\alpha$ expression. PLoS One 8, e68017 (2013).

5. Feingold, K. R. Thematic review series: skin lipids. The role of epidermal lipids in cutaneous permeability barrier homeostasis. J Lipid Res. 48, 2531-2546 (2007).

6. Schurer, N. Y. \& Elias, P. M. The biochemistry and function of stratum corneum lipids. Adv Lipid Res. 24, 27-56 (1991).

7. Feingold, K. R. The regulation and role of epidermal lipid synthesis. Adv Lipid Res. 24, 57-82 (1991).

8. Downing, D. T. Lipid and protein structures in the permeability barrier of mammalian epidermis. J. Lipid Res. 33, 301-313 (1992).

9. Menon, G. K., Brown, B. E. \& Elias, P. M. De novo sterologenesis in the skin.II Regulation by cutaneous barrier requirements. J. Lipid Res. 26, 418-427 (1985)

10. Jackson, S. M. et al. Effect of cutaneous permeability barrier disruption on HMGCoA reductase, $\mathrm{LDL}$ receptor, and apolipoprotein $\mathrm{E}$ mRNA levels in the epidermis of hairless mice. J. Lipid Res. 33, 1307-1314 (1992).

11. Feingold, K. R. et al. Cholesterol synthesis is required for cutaneous barrier function in mice. J. Clin. Invest. 86, 1738-1745 (1990).

12. Ye, J. et al. Alterations in cytokine regulation in aged epidermis: implications for permeability barrier homeostasis and inflammation. I. IL-1 gene family. Exp. Dermatol. 11, 209-216 (2002).

13. Ghadially, R. et al. Decreased epidermal lipid synthesis accounts for altered barrier function in aged mice. J. Invest. Dermatol. 106, 1064-1069 (1996).
14. Valencia, A. \& Kochevar, I. E. Ultraviolet A induces apoptosis via reactive oxygen species in a model for Smith-Lemli-Opitz syndrome. Free Radic. Biol. Med. 40, 641-650 (2006).

15. Epstein, E. H., Williams, M. L. \& Elias, P. M. The epidermal cholesterol sulfate cycle. J. Am. Acad. Dermatol. 10, 866-868 (1984).

16. Denning, M. F., Kazanietz, M. G., Blumberg, P. M. \& Yuspa, S. H. Cholesterol sulfate activates multiple protein kinase $\mathrm{C}$ isoenzymes and induces granular cell differentiation in cultured murine keratinocytes. Cell Growth Differ. 6, 1619-1626 (1995).

17. Morita, T., Yoshiga, K., Takada, K. \& Okuda, K. Effect of a chemical carcinogen and phorbol esters on sterol metabolism of mouse skin. Cancer Res. 41, 2943-2949 (1981).

18. Horton, J. D., Goldstein, J. L. \& Brown, M. S. SREBPs: activators of the complete program of cholesterol and fatty acid synthesis in the liver. J. Clin. Invest. 109, 1125-1131 (2002).

19. Strott, C. A. \& Higashi, Y. Cholesterol sulfate in human physiology: what's it all about? J. Lipid Res. 44, 1268-1278 (2003).

20. Javitt, N. B. et al. Cholesterol and hydroxycholesterol sulfotransferases: identification, distinction from dehydroepiandrosterone sulfotransferase, and differential tissue expression. Endocrinology. 142, 2978-2984 (2001).

21. Kawabe, S. et al. Cholesterol sulfate activates transcription of transglutaminase 1 gene in normal human keratinocytes. J. Invest. Dermatol. 111, 1098-1102 (1998).

22. Hanley, K. et al. Cholesterol sulfate stimulates involucrin transcription in keratinocytes by increasing Fra-1, Fra-2, and Jun D. J. Lipid Res. 42, 390-398 (2001).

23. Hanley, K. et al. Activators of the nuclear hormone receptors PPARalpha and FXR accelerate the development of the fetal epidermal permeability barrier. J. Clin. Invest. 100, 705-712 (1997).

24. Jans, R., Atanasova, G., Jadot, M. \& Poumay, Y. Cholesterol depletion upregulates involucrin expression in epidermal keratinocytes through activation of p38. J. Invest. Dermatol. 123, 564-573 (2004).

25. Furuchi, T. \& Anderson, R. G. Cholesterol depletion of caveolae causes hyperactivation of extracellular signal-related kinase (ERK). J. Biol. Chem. 273, 21099-21104 (1998).

26. Katsanakis, K. D., Owen, C. \& Zoumpourlis, V. JNK and ERK signaling pathways in multistage mouse carcinogenesis: studies in the inhibition of signaling cascades as a means to understand their in vivo biological role. Anticancer Res. 22, 755-759 (2002).

27. Taniguchi, F. et al. Activation of mitogen-activated protein kinase pathway by keratinocyte growth factor or fibroblast growth factor-10 promotes cell proliferation in human endometrial carcinoma cells. J. Clin. Endocrinol. Metab. 88, 773-780 (2003)

28. Samuel, S. \& Bernstein, L. R. Adhesion, migration, transcriptional, interferoninducible, and other signaling molecules newly implicated in cancer susceptibility and resistance of JB6 cells by cDNA microarray analyses. Mol. Carcinog. 39, 34-60 (2004).

29. Kemp, C. J. Multistep skin cancer in mice as a model to study the evolution of cancer cells. Semin. Cancer Biol. 15, 460-473 (2005).

30. Holmer, L., Pezhman, A. \& Worman, H. J. The human lamin B receptor/sterol reductase multigene family. Genomics. 54, 469-746 (1998).

31. Fernandez-Ranvier, G. G. et al. Candidate diagnostic markers and tumor suppressor genes for adrenocortical carcinoma by expression profile of genes on chromosome 11q13. World J. Surg. 32, 873-881 (2008).

32. Williams, M. D. et al. Differential gene expression profiling of aggressive and nonaggressive follicular carcinomas. Hum. Pathol. 42, 1213-1220 (2011).

33. Hanahan, D. \& Weinberg, R. A. The hallmarks of cancer. Cell 100, 57-70 (2000).

34. Hanahan, D. \& Weinberg, R. A. Hallmarks of cancer: the next generation. Cell 144, 646-674 (2011).

35. Andreadis, S. T., Hamoen, K. E., Yarmush, M. L. \& Morgan, J. R. Keratinocyte growth factor induces hyperproliferation and delays differentiation in a skin equivalent model system. FASEB J. 15, 898-906 (2001).

36. Wang, P. Y., Weng, J. \& Anderson, R. G. OSBP is a cholesterol-regulated scaffolding protein in control of ERK 1/2 activation. Science 307, 1472-1476 (2005).

37. Kelley, R. I. \& Herman, G. E. Inborn errors of sterol biosynthesis. Annu. Rev. Genomics Hum. Genet. 2, 299-341 (2001).

38. Brunetti-Pierri, N. et al. Lathosterolosis, a novel multiple-malformation/mental retardation syndrome due to deficiency of 3beta-hydroxysteroid-delta5desaturase. Am. J. Hum. Genet. 71, 952-958 (2002)

39. Cañueto, J., Girós, M. \& González-Sarmiento, R. The role of the abnormalities in the distal pathway of cholesterol biosynthesis in the Conradi-Hünermann-Happle syndrome. Biochim. Biophys. Acta. 1841, 336-344 (2013).

40. Mirza, R. et al. Increased expression of aquaporin-3 in the epidermis of DHCR24 knockout mice. Br. J. Dermatol. 158, 679-684 (2008).

41. He, M. et al. Mutations in the human SC4MOL gene encoding a methyl sterol oxidase cause psoriasiform dermatitis, microcephaly, and developmental delay. J. Clin. Invest. 121, 976-984 (2011).

42. Seeger, M. A. \& Paller, A. S. The role of abnormalities in the distal pathway of cholesterol synthesis in the Congenital Hemidysplasia with Ichthyosiform erythroderma and Limb Defects (CHILD) syndrome. Biochim. Biophys. Acta. 1841, 345-352 (2013). 
43. Zhang, P. et al. The orphan nuclear receptor Nur77 regulates hepatic cholesterol metabolism through the suppression of LDLR and HMGCR expression. Mol. Med. Rep. 5, 1541-1547 (2012).

44. Hubbert, M. L., Zhang, Y., Lee, F. Y. \& Edwards, P. A. Regulation of hepatic Insig-2 by the farnesoid X receptor. Mol. Endocrinol. 21, 1359-1369 (2007).

45. Zhang, Y. et al. Downregulation of human farnesoid X receptor by miR-421 promotes proliferation and migration of hepatocellular carcinoma cells. Mol Cancer Res. 10, 516-522 (2012).

46. Zhang, Y. et al. Loss of FXR protects against diet-induced obesity and accelerates liver carcinogenesis in ob/ob mice. Mol. Endocrinol. 26, 272-280 (2012).

47. Rearick, J. I., Albro, P. W. \& Jetten, A. M. Increase in cholesterol sulfotransferase activity during in vitro squamous differentiation of rabbit tracheal epithelial cells and its inhibition by retinoic acid. J. Biol. Chem. 262, 13069-13074 (1987).

48. Barrett, K. G. et al. Regulation of murine hepatic hydroxysteroid sulfotransferase expression in hyposulfatemic mice and in a cell model of 3'-phosphoadenosine5'-phosphosulfate deficiency. Drug Metab. Dispos. 41, 1505-1513 (2013).

49. Chida, K. et al. Cholesterol sulfate, a second messenger for the eta isoform of protein kinase $\mathrm{C}$, inhibits promotional phase in mouse skin carcinogenesis. Cancer Res. 55, 4865-4869 (1995).

50. Kiguchi, K. et al. Alterations in cholesterol sulfate and its biosynthetic enzyme during multistage carcinogenesis in mouse skin. J. Invest. Dermatol. 111, 973-981 (1998)

51. Lampe, M. A., Williams, M. L. \& Elias, P. M. Human epidermal lipids: characterization and modulations during differentiation. J. Lipid Res. 24, 131-140 (1983).
52. Macala, L. J., Yu, R. K. \& Ando, S. Analysis of brain lipids by high performance thin-layer chromatography and densitometry. J. Lipid Res. 24, 1243-1250 (1983).

\section{Acknowledgments}

This work was supported by PRIN (Progetti di Ricerca di Interesse Nazionale) (20092BE97Y_005), and Fondazione Cassa di Risparmio di Perugia (2013.0215.021 and 2013.0077.021) to RR and IB. The funders had no role in study design, data collection and analysis, decision to publish, or preparation of the manuscript.

\section{Author contributions}

I.B., A.M., R.R. and M.J.P. designed the experiments, interpreted the results and wrote the paper. A.S. and R.dS. performed histochemical analysis. L.G. and I.B. performed the experiments. All authors reviewed the manuscript.

\section{Additional information}

Supplementary information accompanies this paper at http://www.nature.com/ scientificreports

Competing financial interests: The authors declare no competing financial interests. How to cite this article: Bellezza, I. et al. The loss of Tm7sf gene accelerates skin papilloma formation in mice. Sci. Rep. 5, 9471; DOI:10.1038/srep09471 (2015).

This work is licensed under a Creative Commons Attribution 4.0 International License. The images or other third party material in this article are included in the article's Creative Commons license, unless indicated otherwise in the credit line; if the material is not included under the Creative Commons license, users will need to obtain permission from the license holder in order to reproduce the material. To view a copy of this license, visit http://creativecommons.org/licenses/by/4.0/ 